\author{
Military Technical College \\ Kobry El-Kobbah, \\ Cairo, Egypt.
}

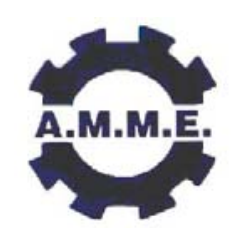

$13^{\text {th }}$ International Conference on Applied Mechanics and Mechanical Engineering.

\title{
DEVELOPMENT OF INDOOR NAVIGATION SYSTEM FOR MOBILE ROBOT BY USING RFID
}

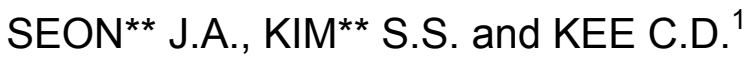

\begin{abstract}
RFID system can be used to improve object recognition, map building and localization for robot area. A novel method of indoor navigation system for a mobile robot is proposed using RFID technology. The mobile robot with a RFID reader and antenna is able to find what obstacles are located where in circumstance and can build the map similar to indoor circumstance by combining RFID information and distance data obtained from other sensors. Using the map obtained, the mobile robot can avoid obstacles and finally reach the desired goal by $A^{*}$ algorithm. 3D map which has the advantage of robot navigation and manipulation is able to be built using $x, y, z$ dimensions of products. The proposed robot navigation system is proved to apply for SLAM and path planning in unknown circumstance through numerous experiments.
\end{abstract}

\section{KEY WORDS}

Mobile Robot, A*, Map Building, Path Planning, Localization, SLAM, 3D Map, Object Recognition

1 Professor, School of Mechanical Systems Engineering, Chonnam. National University, South Korea. E-mail : cdkee@chonnam.ac.kr, TEL : +86-62-530-1667 FAX : + 82-62-530-1689.

** Ph.D. students, School of Mechanical Systems Engineering, Chonnam. National University, South Korea 


\section{INTRODUCTION}

An autonomous navigation is important and key role for intelligent robot to perform its tasks required. Issues in this field include self-localization, path planning, map building, obstacle avoidance, and object recognition. Recently, many studies are under going to solve these problems using RFID [1-10]. The TAG project by AIST(Advanced Industrial Science and Technology) in Japan and the GUIDE project by Intel are the cases that RFID is applied to robots[1,2].

For the ubiquitous age, most of products will be produced with a passive RFID tag instead of bar-code. Therefore, users can acquire the information in tag freely through internet network, regardless of where they are. In this study, we would like to realize autonomous navigation in unknown circumstance with the application of RFID system. With a robot equipped with a RFID reader and antenna, it is proved to obtain a map which is satisfactorily similar to an indoor circumstance based on the application of the map building, object recognition, path planning, and localization algorithms. Finally, the reliability of the proposed algorithm is verified through numerous experiments.

\section{ROBOT NAVIGATION SYSTEM}

\section{System Configuration}

A mobile robot requires basically mobility because it should perform given tasks autonomously in variable circumstances. Robot Navigation System(RNS) is used for robot to estimate its current location to plan the optimal path to a destination avoiding obstacles. RNS proposed in this research is developed for various mobile robots in indoor circumstance. Figure 1 shows the configuration of the proposed RNS system.

Hardware is made up of two stepping motors, four ultrasonic sensors, eight infrared(IR) sensors, RFID system, and a DSP module. The Bluetooth system based on RS-232C is used for communication, and Infinity210 and a passive tag of Sirit Ltd. are used for RFID system.

The display of two windows is developed using Visual C++ 6.0 and OPEN GL. One is a dialog window for components tests of mobile robot, and the other is for map building, localization, and path planning. DSP control dialog shown in Fig.2(a) displays the states of communication, ultrasonic sensors and infrared sensors, and RFID operating results, and Fig.2(b) shows a control dialog for RFID. It contains several functions of saving objects information, function of inputting data that you want to store to the tag, and function of returning $x, y, z$ dimensions. Thus, it can test many fundamental functions about RFID system. Figure 3 shows the main window of RNS. 


\section{A NAVIGATION ALGORITHM FOR MOBILE ROBOTS}

\section{Object Recognition}

It is supposed that in the ubiquitous age, tag-attached products will be arranged in any local circumstance, and we are able to acquire any information including shape or size data through the internet network only with the name of a product.

The way that a robot recognizes an object through RFID technology is as follows. First, the robot recognizes the tag of product, and the reader sends the tag ID to DSP. Then, DSP re-sends it to a PC. The tag ID is compared with many object data stored in the server, and according to the name of the product, required data can be obtained from the PC. Based on this information, the object is located on a map using the distance data obtained from infrared sensors and ultrasonic sensors.

The flow chart of a navigation algorithm is shown in Fig. 4. The applied algorithm divides the criteria into four cases in order to locate an object. These four cases are as follows: First, the case that both $\mathrm{x}$ and $\mathrm{y}$ dimensions of product are identified, second, either $x$ or $y$ is identified, third, it is detected through RFID, but neither $x$ or $y$ is identified, and finally the case that either $x$ or $y$ is identified, and the other is not found. If it doesn't come into any of these four cases, the navigation is not disturbed, so this object is ignored.

\section{Map Building}

In the case that a map is built only with infrared sensors and ultrasonic sensors, only the existence of object is checked. Therefore, a map is constructed only based on the paths which a robot goes through. However, if RFID tag is attached on objects, 3D map can be constructed using the information stored in the tag.

The map building algorithm is composed of three steps, and the flow chart of it is shown in Fig. 5. First, it finds out the size of a map by circling clockwise to find the nearest wall. Through the tags attached on objects, the robot recognizes which objects are where located around. When the first step is completed, it moves inward and circles clockwise for more detailed map building. The robot moves judging whether there is already visited or not. When an object is recognized during searching motion, it is marked on a map. Based on the information after map building, three dimensional map on a window using Open GL is displayed.

\section{Path Planning}

In this study, the $A^{*}$ algorithm is employed in order to find the most efficient path to the target node. The algorithm is realized to search an optimal path and minimize turns and go as straight as possible so that a robot motion looks like more intellectual and natural. The path through aesthetic optimization of the $A^{*}$ algorithm is shown in Fig. 6.

\section{Localization}

The method of localization of a robot in this study is to start at any central point, and 
renews the point considering its direction and distance whenever the robot moves. That is, if any object is recognized on the course, it estimates how far the robot itself is away from the object by calculating the distance between the robot's current point and the object's edge point which is the closest to the robot. After the map building, every distance between robot and each object is calculated, then, robot's location is represented on the map from the nearest object. In Fig. 6, the square in lower part shows how it is displayed.

\section{SIMULATION AND EXPERIMENT}

\section{The Configuration of Experimental Apparatus and Experimental Method}

The experimental apparatus of a mobile robot navigation using RFID is composed of a mobile robot with several sensors and PC. Fig. 7 shows the mobile robot and the tag used in the study, and Fig. 8 shows the RNS program. A mobile robot is produced in a small size. The dimension of the mobile robot is approximately $200 \mathrm{~mm} \times 160 \mathrm{~mm}$, and has the maximum speed of $52 \mathrm{~cm} / \mathrm{sec}$. First of all, the path is generated using $A^{*}$ algorithm, then, confirm whether the robot moves along the generated path. After that, SLAM and path planning are verified as $3 \mathrm{D}$ map is constructing. That is, during the robot navigation, the distance from object is measured by infrared sensors and ultrasonic sensors, and the tag ID on object is recognized by a RFID reader. These data are sent to PC through Bluetooth wireless module, then, map building, localization and path planning are implemented. Finally, the 3D map for the given circumstances is completed using Open GL.

\section{Experimental Environment}

It is assumed that the indoor environment is closed by walls on flat floor, and there are various objects such as a desk, a bookcase, a computer, a box, etc. in this space. Fig. 9 shows a real experimental environment.

\section{Experiment Results}

\section{Path planning and locomotion}

Experiments are conducted with a robot to select the optimal path from a starting point to a destination avoiding obstacles. After virtual experiments through simulation, practical experiments with a robot are conducted to prove its practicability. The simulation is carried out with objects of real size virtually arranged in the space. The result is, as shown in Fig. 10, that the robot selects a path and moves to the destination very well. The result of the practical experiment is shown in Fig. 11, and it is almost the same as that of the simulation.

\section{SLAM, Path planning and 3D mapping in virtual space}

First, computer simulation is conducted to verify whether a mobile robot performs SLAM, path planning, object recognition and 3D map building correctly by using the proposed RNS system. Simulations are conducted in a virtual rectangular parallelepiped space, the length of $7800 \mathrm{~mm}$, the width of $8000 \mathrm{~mm}$, and the height of $200 \mathrm{~mm}$ as the experimental environment. Various objects are also arranged in this 
virtual space. Simulations are carried out in two ways. One is with tags attached on objects, and the other without tags. In the simulation, SLAM and path planning are performed based on the information of object data from RFID and distance data from range sensors virtually established in the space. Finally, it builds a three-dimensional map using Open GL.

The results of the simulation in virtual indoor space are shown in Fig. 12. In the case that RFID tags are not used, the robot recognizes the objects as just obstacles, and can not get the information of $A, B$ areas in virtual space, therefore an incorrect map which is much different from the indoor space is built. That is, a map which shows the minimal areas that the robot can go through is composed. However, in the case that RFID tags are used, a map which is very similar to the virtual indoor circumstance is obtained, and also a $3 \mathrm{D}$ map that is able to be used for robot manipulation and navigation is built using the information of the detected objects.

\section{SLAM, path planning and 3D map building in real space}

Numerous experiments are conducted to verify the reliability of the simulation. The experiments are also carried out in two ways, one with RFID tags attached on objects, and the other without the tags. Fig.13 shows the results of the experiments without RFID tags, and we recognize that the two maps obtained from experiment and simulation respectively are not matched acceptably, because of the step motor errors and a standardized grid size of $200 \times 200 \mathrm{~mm}$ on the floor.

The results of the experiments with RFID tags on objects are shown in Fig. 14. In this case, we also can not get the map which is matched well to the real environment. However, combining the objects information obtained from RFID tags and distance data from ultrasonic sensors and infrared sensors, more precise map is constructed. Finally, this map is proved to be used for robot navigation by planning a path avoiding randomly located obstacles.

\section{CONCLUSION}

In this study, a Robot Navigation System using RFID and Simulation Program for autonomous navigation are proposed. The results of the study are as follows:

(1) Certain information like dimension or shape of objects detected in given space using RFID tags is verified to build 3D-map, which is used for robot localization and path planning avoiding obstacles.

(2) Combining the shape information from RFID system and the distance data from ranger sensors, a 3D-map which is much similar to the real circumstance is constructed.

(3) The reliability of the developed simulation program for efficient localization and path planning for a mobile robot is demonstrated through numerous experiments.

\section{REFERENCES}

[1] Chong, N.Y., Hongu, H., Ohba, K., Hirai, S. and Tanie, K., "A distributed knowledge network for real world robot application," Proc. IEEE/RSJ Int. Conf. 
Intelligent Robots and Systems, pp. 187-192, (2004).

[2] Philipose, M., Fishkin, K., Fox, D., Katz, H., Patterson, D. and Perkowitz, M., "Guide: Toward understanding daily life via auto-identification and statistical analysis," In proc. of the Int. Workshop on Ubiquitous Computing for Pervasive Healthcare Applications(Ubi-heath), (2003).

[3] Kantor, G. and Sinha, S., "Preliminary Results in Range-Only Localization and Mapping," IEEE Int. Conf. Robotics and Automation, pp. 1818-1823, (2002).

[4] Tsukiyama, T., "Global Navigation System with RFID Tags", Proc. SPIE, Vol.4573, pp.256-264, (2002).

[5] Hahnel, D., Burgard, W., Fox, D., Fishkin, K. and Philipose, M., "Mapping and localization with RFID technology," IEEE Int. Conf. Robotics and Aumation, pp. 1015-1020, (2004).

[6] Yamano, K., Tanaka, K., Hirayama, M., Kondo, E., Kimuro, Y. and Matsumoto, M., "Self-localization of mobile robots with RFID system by using support vector machine," IEEE Int. Conf. Intelligent Robots and Systems, pp. 3756-3761, (2004).

[7] Chong, N.Y., Hongu, K., Miyazaki, M. and Takemura, K., "Robots on Self-Organizing Knowledge Networks", Int. Proc. ICRA 2004. pp.3494-3499, (2004).

[8] Boukraa, M. and Ando, S., "A computer vision system for knowledge-based 3D scene analysis using radio-frequency tags", Multimedia and Expo, 2002. ICME '02 Proceedings. 2002 IEEE International Conference on, Vol.2, pp.245-248, (2002).

[9] Lee, H.J. and Lee, M.C., "Localization of Mobile Robot Based on Radio Frequency Identification Devices", SICE-ICASE International Joint Conference, pp 5934-5939, (2006).

[10] Seo, D.S., Lee, H.G., Kim, H.S., Yang, G.W. and Won, D.H., "Monte Carlo Localization for Mobile Robots Under RFID Tag Infrastructures", Journal of Control, Automation, and Systems Engineering Vol. 12, No. 1, pp 47-53, (2006). 


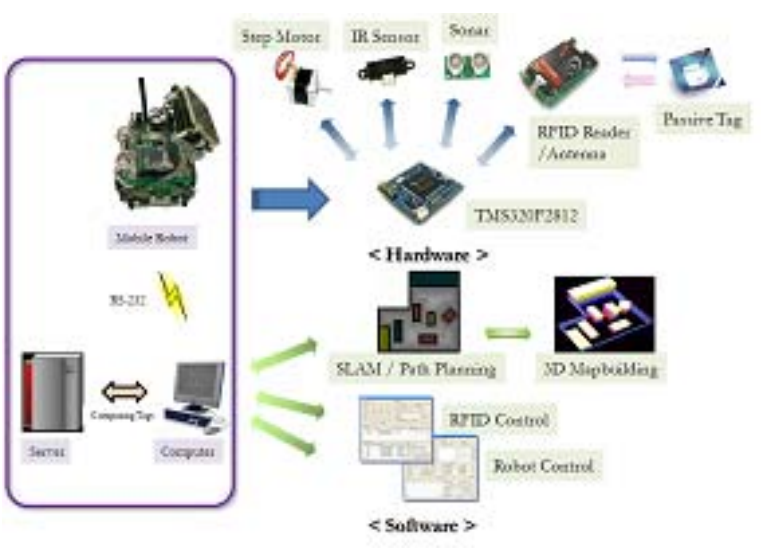

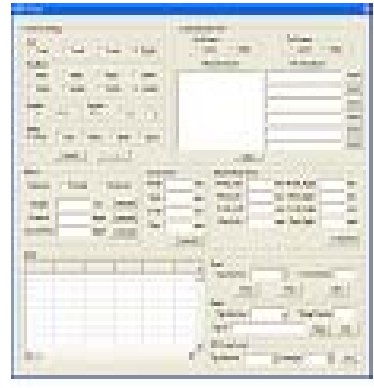

(a) DSP test dialog

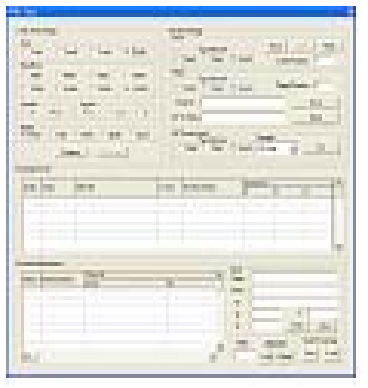

(b) RFID test dialog

Fig. 2 Control dialog

Fig. 1 Schematic diagram of RNS

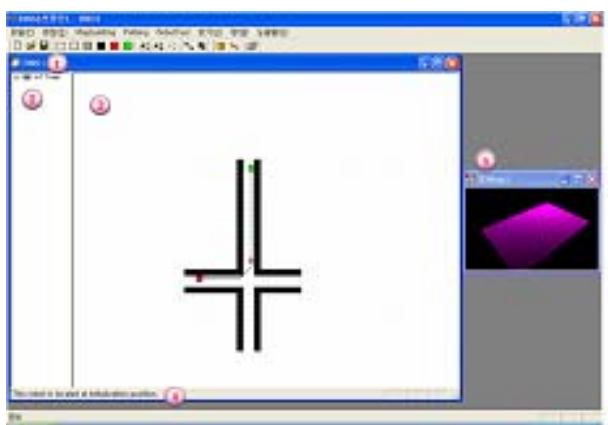

Fig. 3 Main window of RNS

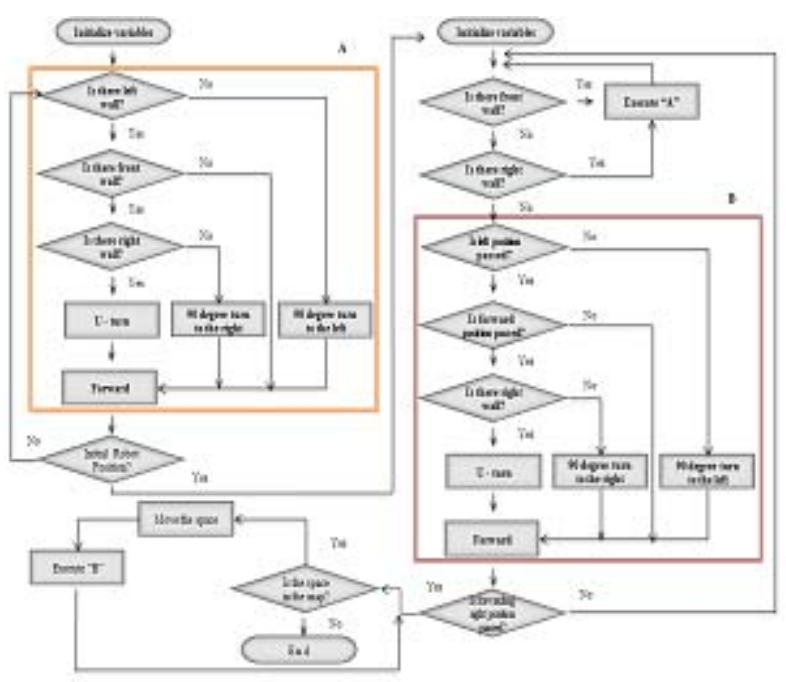

Fig. 5 Flow chart of map building algorithm.

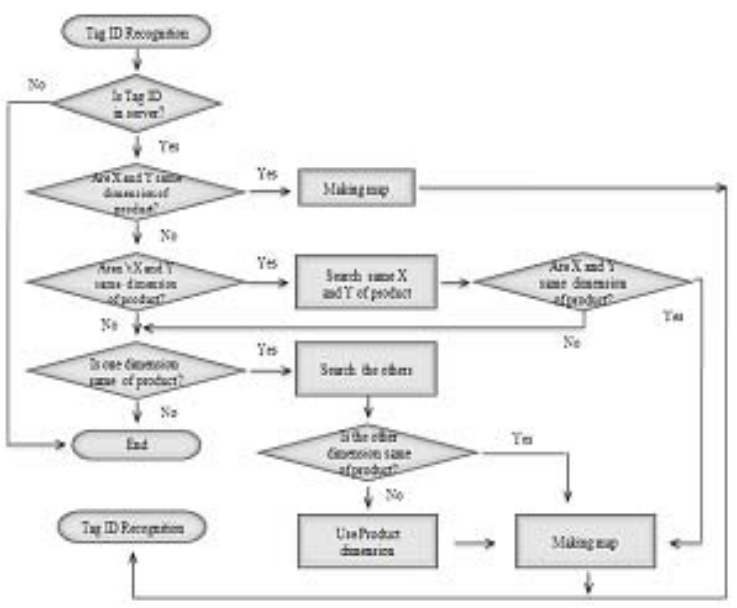

Fig. 4 Flow chart of product recognition algorithm

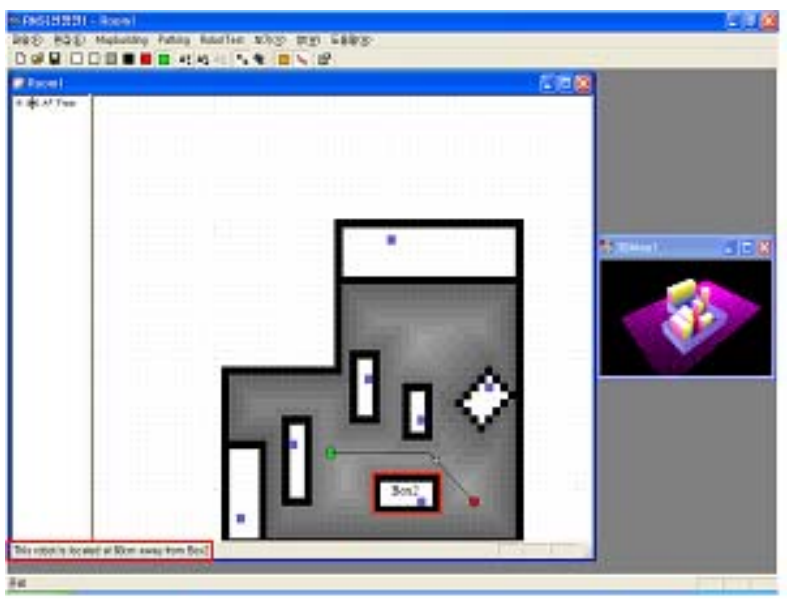

Fig. 6 A example of path planning and localization. 


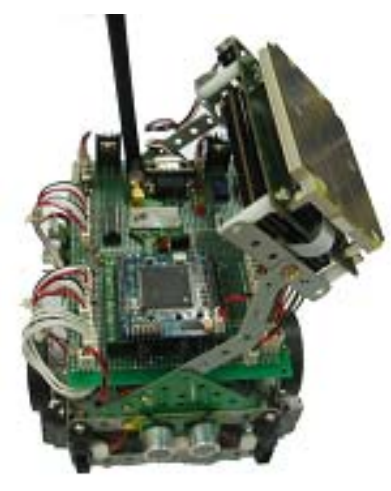

Fig. 7 The developed mobile robot

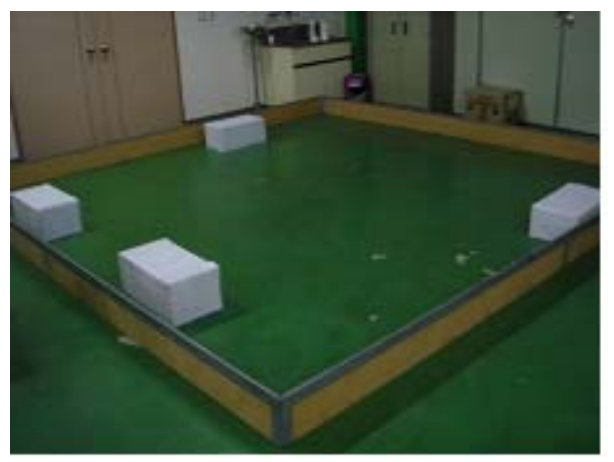

Fig. 9 Experimental environment
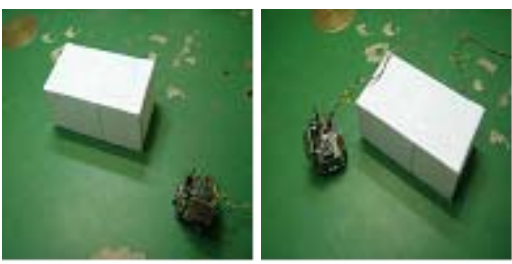

(a) Start point

(b) In progress

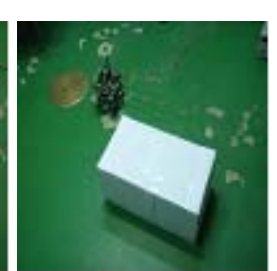

(c) End point

Fig. 11 Experiment result of Path planning

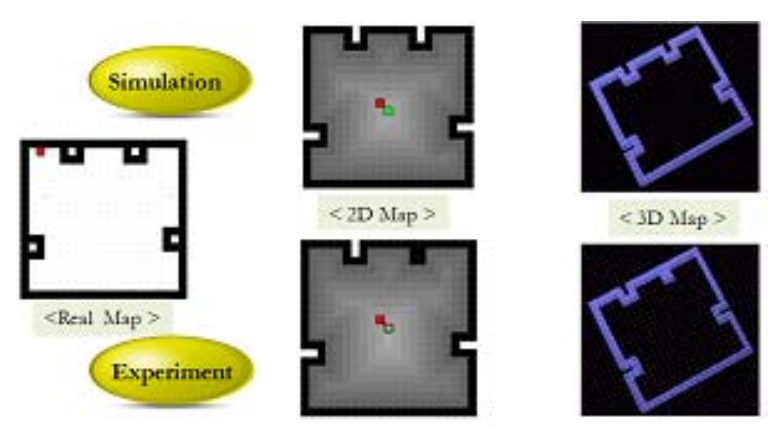

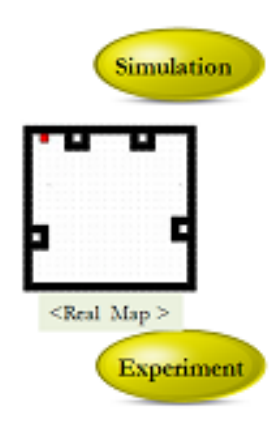

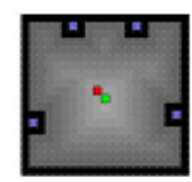

(a) Start point

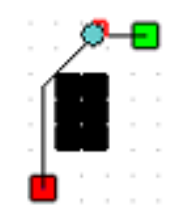

(b) In progress

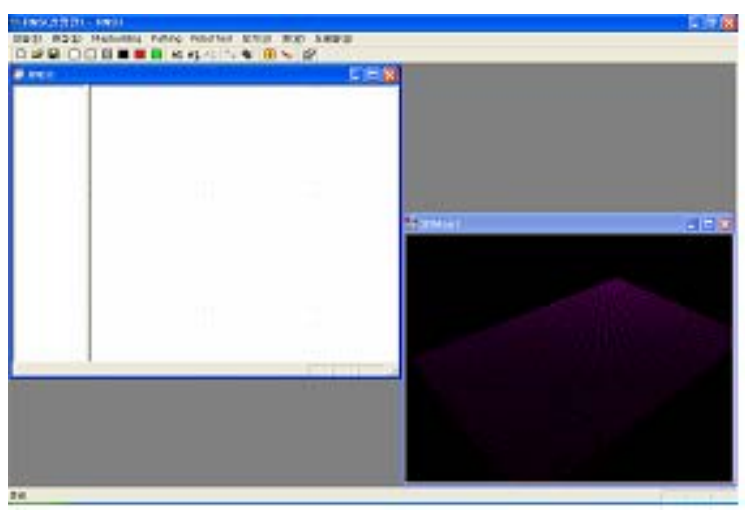

Fig. 8 Main window of developed RNS

Fig. 10 Simulation result of Path planning

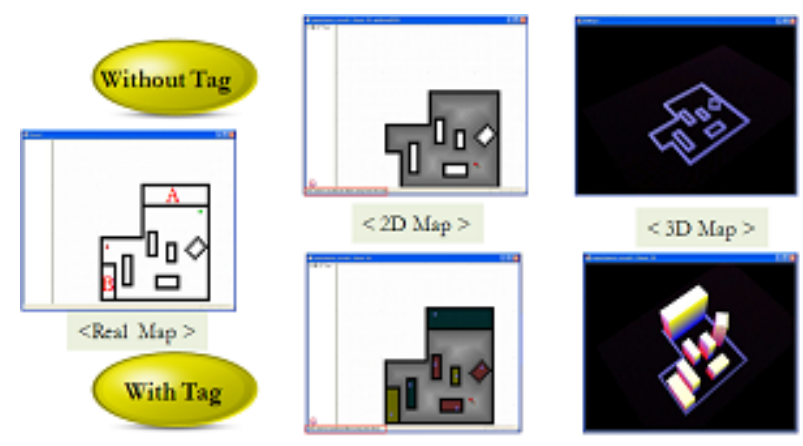

Fig. 12 Simulation results in virtual space

Fig. 14 Simulation and experiment results with tags.

Fig. 13 Simulation and experiment results without tags. 